\title{
Effect of Home-based Exercise Therapy for Peripheral Arterial Disease Patients Underwent Endovascular Treatment: A Clinical Controlled Design
}

\author{
Shota Otsuka, Msc, $\mathrm{PT}^{1}$, Tomoyuki Morisawa, Ph.D, PT², Yu HoJo, $\mathrm{PT}^{1}$, \\ Atsuhisa IsHIDA, Ph.D, Dr $^{3}$ and Akira TAMAKI, Ph.D, $\mathrm{PT}^{4}$ \\ ${ }^{1)}$ Department of Rehabilitation, the Sakakibara Heart Institute of Okayama, Japan \\ ${ }^{2}$ Department of Physical Therapy, Juntendo University, Japan \\ ${ }^{3)}$ Department of General Surgery, Kawasaki Medical School General Medical Center, Japan \\ ${ }^{4)}$ Department of Rehabilitation, Hyogo University of Health Sciences, Japan
}

\begin{abstract}
Objective: This study aimed to clarify the effect of home-based exercise therapy on physical activity in peripheral arterial disease (PAD) patients after EVT. Methods: Study design was controlled clinical design. The subjects were 30 patients $(76.6 \%$ men) who underwent EVT in the Sakakibara Heart Institute of Okayama. Patients with EVT meeting the inclusion criteria were divided into two groups, intervention group (Home-based exercise) and control group. Patients' basic characteristics, the number of steps walked and QOL questionnaire (WIQ, SEPA, Vascu QOL) were assessed before surgery and, at the 3 month after discharge. A two-way analysis of variance (ANOVA) was performed to compare number of steps walked and QOL questionnaire. Results: Interaction effect were observed in the number of steps walked (F $(\mathbf{1 , 2 8})=13.89, \mathrm{p}<0.01)$. A multiple comparison test confirmed a significant increase between results of before surgery and at three months after surgery in the intervention group $(\mathbf{p}<\mathbf{0 . 0 1})$. An interaction between the presence and absence of intervention was found for the WIQ pain score $(F(1,28)=5.86, p=0.01)$, speed score $(\mathrm{F}(1,28)=3.80, \mathrm{p}=0.04)$ and $\operatorname{SEPA}(\mathrm{F}(1,28)=4.99, \mathrm{p}=0.03)$. In a multiple comparison study, there was a significant increase in WIQ pain and speed scores in both groups before and 3 months after discharge from the hospital. Conclusion: Home-based exercise therapy using physical activity indices has the potential to improve number of steps and quality of life in patients with PAD after EVT.
\end{abstract}

Key words: Home-based exercise, Peripheral arterial desease, Endovascular treatment, Physical activity

(Phys Ther Res 24: 120-127, 2021)

$\mathbf{P}$ pending on the severity of the condition. Endovascular treatment $(E V T)$ is the first choice for patients with symptomatic PAD in the Transatlantic Inter-Society Consensus (TASC) classification ${ }^{1)}$. In addition, EVT is a minimally invasive and safe treatment method that is rapidly increasing $^{2)}$. However, due to its benefits, many patients have a

Received: June 13, 2020

Accepted: November 27, 2020

Advance Publication by J-STAGE: February 24, 2021

Correspondence to: Shota Otsuka, Department of Rehabilitation, the Sakakibara Heart Institute of Okayama, Japan, 2-5-1 Nakai-cho, Kitaku, Okayama-city, Okayama 700-0804, Japan

\# e-mail: qqqz3sm9k@gmail.com

doi: 10.1298/ptr.E10056 short hospital stay and do not receive adequate exercise during their hospital stay. In our previous study, the combination of EVT and exercise therapy during hospitalization resulted in a significant $286 \%$ improvement in maximum walking distance, but not a significant $127 \%$ improvement in number of steps ${ }^{3)}$. This report showed difficulty in obtaining a regular exercise routine after EVT.

PAD patients have a lower amount of physical activity, compared to healthy people. Previous studies have reported that the average number of steps within daily life for PAD patients with intermittent claudication is 3000-4000 steps/day ${ }^{4-6)}$. Patients with low physical activity in PAD are also at higher risk for worsening PAD and developing cardiovascular events ${ }^{7)}$. Garg et $\mathrm{al}^{8)}$ examined cardiovascular mortality at 57 months in four groups of patients with PAD 
according to physical activity level. It was reported that the group with the lowest physical activity level had twice the cardiovascular mortality rate than the group with the highest physical activity level. Therefore, it has been suggested that the management of physical activity is extremely important for patients with PAD in terms of prognosis, including PAD exacerbations and cardiovascular mortality ${ }^{9-12}$. Furthermore, it has been reported that increased physical activity affects the promotion of quality of life in patients with $\mathrm{PAD}^{13}$, suggesting that there is an important relationship between physical activity and quality of life.

For this reason, home-based exercise therapy has recently been attracting attention as a treatment method for patients with PAD. Daily recording, quantification of physical activity using a triaxial accelerometer, strict setting of exercise intensity, and frequent feedback are necessary for the success of home exercise therapy in patients with $\mathrm{PAD}^{14,15)}$. McDermott et al ${ }^{16)}$ compared the control group with the home-based exercise group and reported that at 6 months, the home-based exercise group improved physical activity by $11 \%$, WIQ distance score by $34 \%$, and WIQ speed score by $32 \%$ from baseline.

Despite the above reports, no studies have reported the effect of home-based exercise on physical activity in PAD patients after EVT, which has been on the rise in recent years. This study aimed to clarify the effect of home-based exercise therapy on physical activity in PAD patients after EVT.

\section{Methods}

\section{Trial design}

This study was a prospective, single-center, two-arm, controlled clinical design. In the present study, the eligibility criteria for patients with PAD were those who underwent EVT according to the TASC classification. 84 consecutive PAD patients who underwent EVT between August 2016 and December 2017 participated in the protocol of this study. The TASC classification is defined by the location and distribution of the lesion, which may result in bias in patient performance. Therefore, based on previous studies, the exclusion criteria for this study were established as follows ${ }^{14,15)}$. The following exclusion criteria were applied: (1) unable to walk independently before EVT; (2) diagnosis of dementia; (3) lower extremity amputation; (4) critical limb ischemia; (5) not responding to the postdischarge survey; and (6) missing data.

\section{Outcome}

The primary outcome of the study was the change in the number of steps from pre-EVT to 3 months after discharge from the hospital. Secondary outcomes included changes in the following parameters: WIQ, SEPA and Vascu QOL.

\section{Measurement of physical activity and analysis method}

Physical activity was measured using a triaxial accelerometer (Active style Pro HJA-750C, Omron Co. Japan). A triaxial accelerometer was worn daily from the time of the outpatient visit when the patient was diagnosed with the need for surgery until three months after discharge from the hospital (excluding the hospitalization period), and the number of steps walked was recorded. Patients were instructed to wear a triaxial accelerometer on their lower back throughout the day, from waking up to bedtime, except when bathing or going to bed. The average number of steps was calculated from data that excluded days in which wear time was less than 480 minutes/day for any reason ${ }^{17)}$. The percentage change in steps walked before and at 3 months after surgery was calculated so that individual differences in step count could be taken into account and the effectiveness of home exercise could be evaluated. The following formula was used to calculate the rate of change: (average steps at 3 months after discharge minus average preoperative steps) / x 100.

\section{Evaluation of $Q O L$}

The following questionnaires were measured before hospitalization and at 3 months post-discharge; Walking Impairment Questionnaire (WIQ) ${ }^{18)}$ to evaluate the ability to walk; Self-Efficacy for Physical Activity (SEPA) ${ }^{19)}$ to evaluate subjective self-efficacy; Vascular Quality of Life Questionnaire (Vascu QOL) ${ }^{20)}$ to evaluate ADL of patients with PAD. For the post-discharge quality of life assessment, the questionnaire was mailed to the patient and the patient completed the questionnaire and returned it to us.

\section{Evaluation hemodynamics, exercise tolerance and physical function}

The following items were measured before and after admission and before discharge for pre- and post-EVT assessment. Ankle Brachial Pressure Index (ABI) to evaluate hemodynamics; 6-minute walk distance (6MWD) to evaluate exercise tolerance; Short Physical Performance Battery (SPPB) to evaluate physical function. 6MWD was measured according to the American Thoracic Society (ATS) guidelines $^{21)}$ before EVT and before hospitalization. Patients were asked to walk back and forth for 6 minutes with assistance between two markers placed at $30 \mathrm{~m}$ intervals on flat ground, and their walking distance was measured.

\section{Rehabilitation intervention after endovascular treatment (exercise therapy and pre-discharge instructions)}

After EVT, all patients began standing and walking exercises the day after surgery. When the patient was able to walk independently on level ground without developing complications, exercise therapy with a treadmill was started. One treadmill exercise regimen consisted of a warm-up, a 20-minute treadmill walk, and a cooling down. 


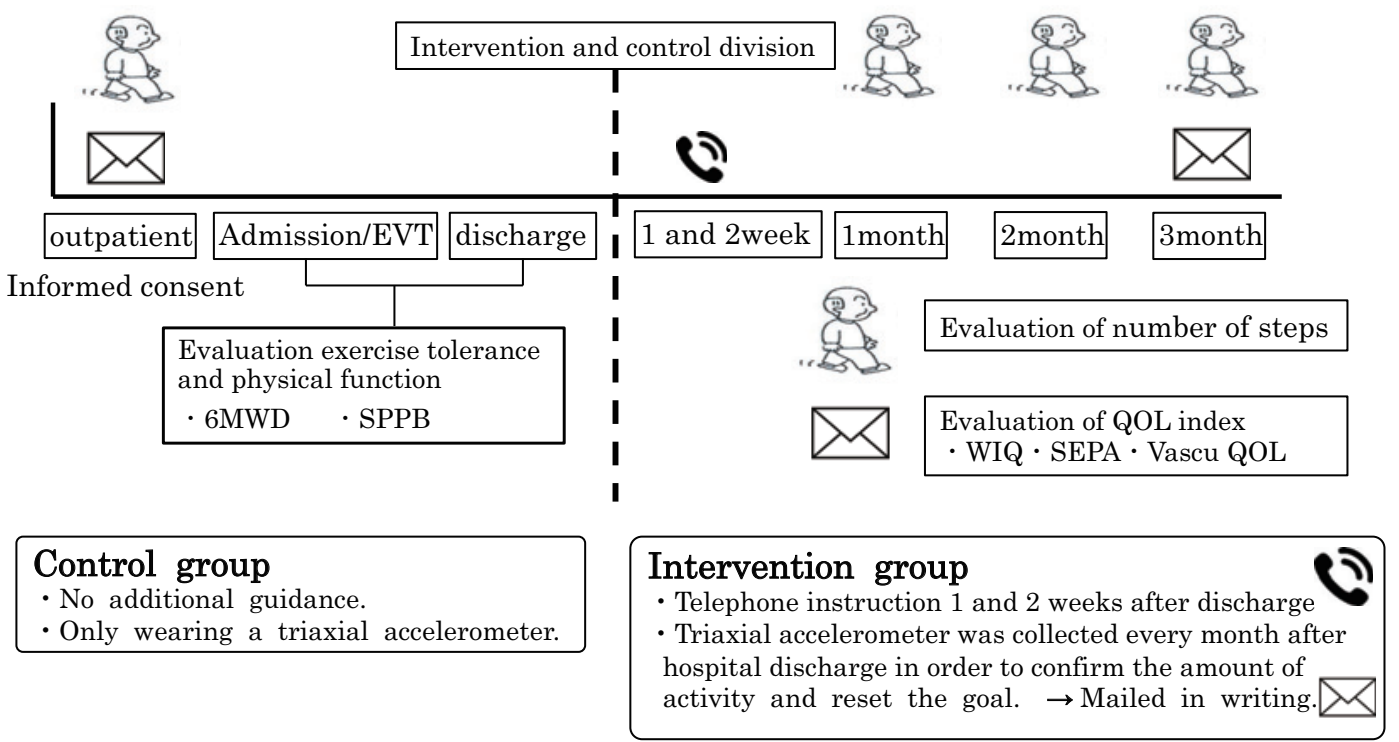

Fig. 1. study protocol

Table 1. Instruction for intervention group

\begin{tabular}{ll}
\hline Walking steps & (1) Increase of 1300 steps/day. \\
(guidelines of Physical Activity and Exercise by MHLW) & (2) Mean number of steps walked in each age bracket. \\
& (3) Goal of older patients. Male 6700 steps, Female 5900 steps. \\
& $\begin{array}{l}\text { ※ Goal was achieved shift to next aim. } \\
\text { Time }\end{array}$ \\
Walking method & - Do comfortable walking speed \\
& - The patient should stop walking when claudication pain is considered moderate (over Borg scale 15).
\end{tabular}

This was performed while the patient was in the hospital. The exercise prescription for treadmill-based exercise therapy was in line with the Trans-Atlantic Inter-Society Consensus II (TASC II) ${ }^{1)}$, an international treatment guideline for PAD. The walking speed and load were gradually increased as the ability to walk improved. The mean number of days of exercise therapy was 5.32 days (range: 2-10 days). At discharge, all patients were instructed to aim to exercise at least three times a week for at least 30 minutes each time. Prior to discharge, the patient received regular exercise advice from the doctor, medication instructions from the pharmacist, and lifestyle guidance from the public health nurse, as well as ADL guidance to avoid excessive flexion at the stent insertion site. For the exercise therapy provided up to the day of discharge and the ADL instruction provided on the day of discharge, the control and intervention groups received the same level of therapeutic intervention. No patients in this study received outpatient rehabilitation after discharge from the hospital.

\section{Randomization}

A controlled clinical trial was used for this study. Patients were alternately assigned to the control and intervention groups.
Protocol in the control group and the intervention group

The exercise protocol for both groups is shown in Figure. 1. The follow-up period was 3 months after discharge from the hospital. After discharge, the protocol did not include additional instruction in the control group. They were also instructed to wear a triaxial accelerometer only. On the other hand, in the intervention group, the post-discharge protocol included instruction based on pre-admission steps and the Ministry of Health, Labour and Welfare's physical activity and exercise guidelines ${ }^{22)}$ (Table 1). The patient was instructed on the number of steps, the duration of walking, and how to walk. The initial goal was to increase the number of steps from the average number of steps taken before admission to the hospital to 1300 steps per day. Each time the goal was achieved, the goal was re-set based on the average number of steps in each age group and the target number of steps for the elderly. Based on previous studies $^{11,12,23)}$, feedback was provided by telephone one to two weeks after discharge from the hospital, and a triaxial accelerometer was collected and results mailed every month after surgery. During the telephone consultations, we reviewed the advice received from other professionals prior to discharge and listened to whether they were practicing the advice and confirmed that they were continuing to exer- 


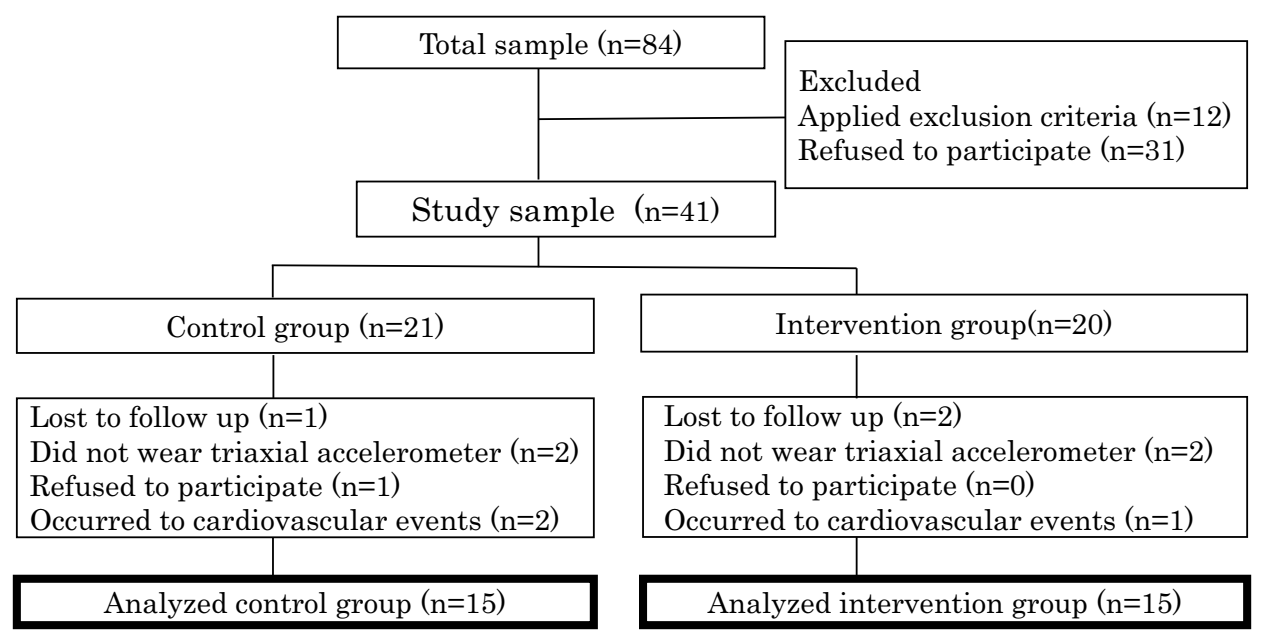

Fig. 2. study flowchart

cise. At the monthly mailing instruction, the triaxial accelerometer received was analyzed and the difference between the average and target steps was presented and the next goal was re-established. All interventions were also carried out by the same physiotherapist. As an evaluation of the implementation of exercise therapy, the rate of achievement of the initial goal at 3 months post-discharge was presented. The initial goal was an increase of 1300 steps/day from the mean number of steps before EVT, as described above.

\section{Analytical methods and statistical methods}

We used unpaired t-tests to compare the averages of continuous variables (such as age) and $\chi^{2}$ tests to compare the proportions of categorical variables (such as sex) between the groups. Rates of change and median were compared using Wilcoxon rank-sum tests. Comparisons of ABI, 6MWD, and SPPB scores before and after EVT were performed using within-groups paired t-test and betweengroups unpaired t-test. Comparisons between changes in number of steps and WIQ, SEPA, Vascu QOL were made using a two-way analysis of variance (ANOVA); if interactions were present, a multiple comparison test was used. In this study, the effect size was calculated in order to evaluate the magnitude of the difference. The $\phi$ coefficient was calculated as the effect size by the $\chi^{2}$ test, and it was interpreted that $\phi=0.1$ is small, $\phi=0.3$ is medium, and $\phi=0.5$ is large. The unpaired and paired t-tests calculated Cohen's $\mathrm{d}$ and interpreted that $\mathrm{d}=0.2$ was small, $\mathrm{d}=0.5$ was medium, and $d=0.8$ was large. For Wilcoxon rank sum test and Mann-Whitney $U$ test, $r$ was calculated and interpreted as $r$ $=0.1$ is small, $r=0.3$ is medium, and $r=0.5$ is large. For twoway analysis of variance, $\eta^{2}$ was calculated and $\eta^{2}=0.01$ was small, $\eta^{2}=0.06$ was medium, and $\eta^{2}=0.14$ was large. All statistical analyses were conducted using IBM SPSS Statistics for Windows, Version 21.0 (IBM Corp., Armonk, $\mathrm{NY}$ ). The threshold for significance was $\mathrm{p}<0.05$.

\section{Ethical considerations}

This study was conducted in accordance with the Helsinki Declaration and was approved by the ethical committee of Hyogo University of Health Sciences (approval number: 16021) and the ethical committee of the Sakakibara Heart Institute of Okayama (approval number: 20140901).

\section{Results}

\section{Study population}

After the study criteria were applied, the remaining 41 patients participated in the study and 41 patients were alternately assigned to the control $(n=21)$ and intervention $(n=$ 20) groups. Among them, 15 in the control group and 15 in the intervention group, who were able to continue wearing the triaxial accelerometer 3 months after surgery, proceeded to analysis (Fig. 2).

Comparison of basic information between the control group and the intervention group

There were no deaths during hospitalization. All patients were discharged home after surgery, with a mean hospital stay of 6.8 days (range: 2-12 days). The mean number of days to triaxial accelerometer data collection was 15.2 days before admission (range, 7-30) and 58.6 days 3 months after discharge (range, 30-84). A comparison of the basic information between the control and intervention groups is shown in Table 2. There was no significant difference between the two groups in terms of basic information and past medical history.

Pre-surgery and post-surgery comparison of the therapeutic areas, hemodynamics, exercise tolerance and physical function between the control group and the intervention group

A comparison of the treatment area, hemodynamics, exercise tolerance and physical function of the control and 
Table 2. Comparison of patient's characteristics between the control group and the intervention group

\begin{tabular}{lcccc}
\hline & Control group $(\mathrm{n}=15)$ & Intervention group $(\mathrm{n}=15)$ & $\mathrm{p}$ value & Effect size \\
\hline Average age (range) & $74.4(66-81)$ & $73.2(70-80)$ & 0.48 & 0.27 \\
Sex (male/female) * & $12 / 3$ & $11 / 4$ & 0.67 & 0.08 \\
BMI (AVG \pm SD) & $24.0 \pm 3.8$ & $22.6 \pm 2.9$ & 0.31 & 0.41 \\
Smoking* & $13(86.7)$ & $14(80.0)$ & 0.54 & 0.11 \\
Fontaine classification (stage II/stage III) & $14 / 1$ & $13 / 2$ & 0.54 & 0.11 \\
Past history n (\%) & & & & 0.27 \\
Hypertension* & $15(100.0)$ & $13(86.7)$ & 0.14 & 0.27 \\
Dyslipidemia* & $15(100.0)$ & $13(86.7)$ & 0.14 & 0.07 \\
Chronic kidney disease* & $8(53.3)$ & $9(60.0)$ & 0.71 & 0.07 \\
Diabetes mellitus* & $8(53.3)$ & $9(60.0)$ & 0.71 & 0.07 \\
Coronary artery disease* & $10(66.7)$ & $9(60.0)$ & 0.70 & 0.07 \\
Cerebral vascular disease* & $4(26.7)$ & $5(33.3)$ & 0.69 & 0.07 \\
Orthopedic disorder* & $5(33.3)$ & $4(26.7)$ & 0.69 & 0 \\
\hline
\end{tabular}

BMI; Body mass index, $*: \chi^{2}$ test

intervention groups is shown in Table 3. There was no significant difference between the two groups in terms of therapeutic area. Significant improvements in ABI were observed within both groups, but there were no significant differences between the two groups. There was no significant difference in the rate of change in 6MWD between the two groups. Similarly, there was no significant difference in SPPB between the two groups.

Pre-surgery and post-surgery comparison of the number of steps walked and the rate of change in the number of steps walked

Interaction effects were observed with the number of walking steps and with or without intervention $(\mathrm{F}(1,28)=$ $13.89, \mathrm{p}<0.01)$. In a multiple comparison test, a significant increase was observed between before surgery and at three

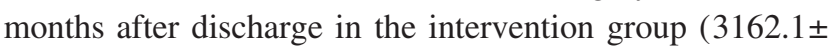
1439.9 vs. $4503.7 \pm 1768.6$; p<0.01) (Fig. 3). In addition, a significantly high rate of change in the number of steps walked was observed in the intervention group at three months after discharge $(13.4 \%$ vs. $46.5 \%$; $<<0.01)$. Initial goal attainment was significantly higher in the intervention group $(20.0 \%$ vs $53.3 \%$; $<<0.01)$.

\section{Pre-surgery and post-surgery comparison of QOL indexes}

An interaction between the presence and absence of intervention was found for the WIQ pain score $(\mathrm{F}(1,28)=$ 5.86, $\mathrm{p}=0.01)$, speed score $(\mathrm{F}(1,28)=3.80, \mathrm{p}=0.04)$ and SEPA $(F(1,28)=4.99, p=0.03)$ (Table 4). In a multiple comparison study, there was a significant increase in WIQ pain and speed scores in both groups before and 3 months after discharge from the hospital.

Between the two groups, the WIQ pain score and speed score were significantly increased in the intervention group 3 months after surgery. (Pain score: $61.6 \pm 22.1$ vs $83.3 \pm 21.7, \mathrm{p}<0.05$; speed score: $55.2 \pm 20.5$ vs $70.4 \pm 16.8$,
$<0.05)$. A significant increase in SEPA was found only in the intervention group before and 3 months after discharge $(42.6 \pm 27.6$ vs $53.8 \pm 25.4 ; \mathrm{p}<0.05)$.

\section{Discussion}

A comparison of the primary outcome of this study, the number of steps, showed a significant difference between the intervention group at the preoperative and 3 months after discharge. In addition, when the rate of change in the number of steps taken before and 3 months after discharge was compared between the two groups, a significantly higher value was observed in the intervention group. Previous studies have shown mixed results on the effects of short-term home exercise therapy on physical activity in patients with PAD. McDermott et $\mathrm{al}^{14)}$ reported that after 6 months of home-based exercise for PAD patients with intermittent claudication, there was an $11 \%$ increase in physical activity from baseline and a significant improvement compared to the control group. However, Gardner et $\mathrm{al}^{15)}$ reported that after 3 months of home-based exercise in patients with PAD, the number of steps increased by $10 \%$ from baseline, but there was no significant improvement compared to the control group and supervised exercise therapy group. In the present study, after 3 months of homebased exercise, the rate of change in the number of steps was significantly increased compared to the control group. he results may be related to two novelties in this study. The first is that the study's population was limited to EVT patients. Patients with PAD with low PA have been reported to be due to females, low education, high comorbidity, low ABI, low motor endurance, and low WIQ score ${ }^{24-26)}$. In this study, the improvement in ABI and WIQ scores was observed with EVT revascularization, suggesting that home exercise may have been more effective than in previous studies. The second factor was setting specific goals for the 
Table 3. Comparison of patient's therapeutic areas, hemodynamics, exercise tolerance and physical function between the control group and the intervention group

\begin{tabular}{|c|c|c|c|c|}
\hline & $\begin{array}{l}\text { Control group } \\
\qquad(\mathrm{n}=22)\end{array}$ & $\begin{array}{l}\text { Intervention group } \\
\qquad(\mathrm{n}=20)\end{array}$ & $\mathrm{p}$ value & Effect size \\
\hline \multicolumn{5}{|l|}{ Therapeutic regions $*(n=42)$} \\
\hline Iliac artery area/Femoral artery area* & $14 / 8$ & $13 / 7$ & 0.93 & 0.01 \\
\hline $\mathrm{ABI}$ & $(\mathrm{n}=15)$ & $(\mathrm{n}=15)$ & & \\
\hline Before EVT $(\mathrm{AVG} \pm \mathrm{SD})$ & $0.66 \pm 0.13 \square$ & $0.72 \pm 0.15 \square+$ & 0.29 & 0.43 \\
\hline After EVT (AVG \pm SD) & $1.02 \pm 0.11 \longrightarrow$ & $0.98 \pm 0.13 \longrightarrow$ & 0.74 & 0.33 \\
\hline 6MWD & $(n=15)$ & $(\mathrm{n}=15)$ & & \\
\hline Before EVT & $292.0(120.0-480.0)$ & $321.6(200.0-460.0)$ & & \\
\hline After EVT & $342.8(220.0-550.0)$ & $372.5(250.0-520.0)$ & & \\
\hline Change of rate $(\%)$ & 117.4 & 115.8 & 0.67 & 0.08 \\
\hline SPPB & $(n=15)$ & $(n=15)$ & & \\
\hline Before EVT median (range) & $12(7-12)$ & $12(7-12)$ & 0.56 & 0.08 \\
\hline After EVT median (range) & $12(8-12)$ & $12(8-12)$ & 0.68 & 0.07 \\
\hline
\end{tabular}

※ There are overlapping therapeutic regions.

ABI; ankle brachial index, EVT; endovascular treatment, 6MWD; 6-minutes walk distance, SPPB; short physical performance battery

$*: \chi^{2}$ test, $\dagger$ : paired t-test vs Before EVT $\mathrm{p}<0.01$

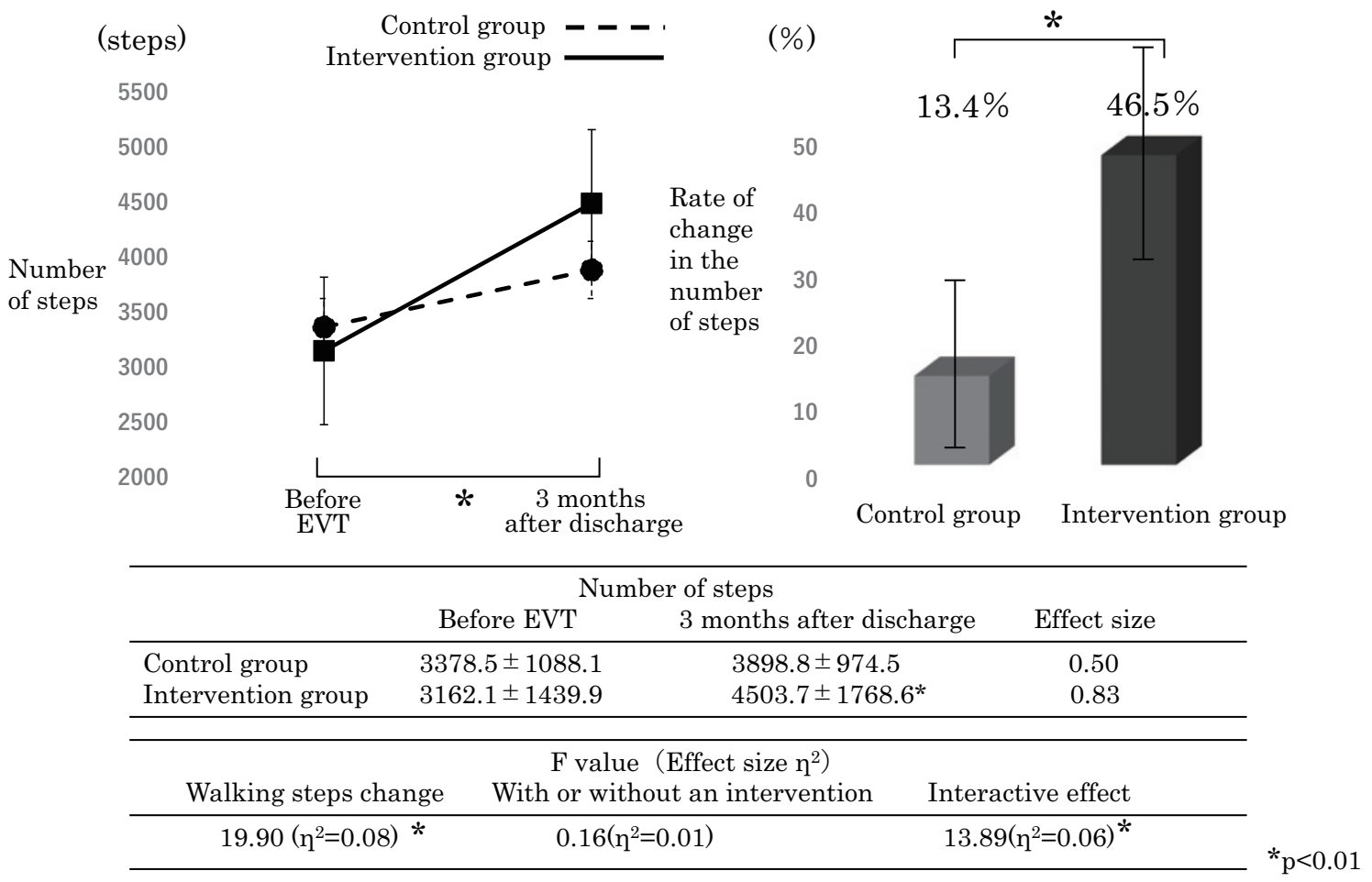

Fig. 3. Comparison of number of steps and rate of change in the number of steps EVT; endovascular treatment

number of steps and periodically rescheduling the goals. The rate of achievement of the initial goal (1300 steps/day increase from the mean number of steps before EVT) was significantly higher in the intervention group. Because previous studies have shown that EVT, inpatient exercise therapy, and discharge instruction alone did not lead to an increase in steps, regular tele-teaching may have been effec- tive in motivating subjects to exercise walking. The content of the guidance used in the intervention group in the current study was not a goal developed specifically for patients with PAD, but was originally developed by our team based on the physical activity and exercise guidelines of the MHLW $^{22}$. Therefore, although the relevance of the instruction is unclear, the team believes that the team has been 
Table 4. Comparison of patient's QOL indexes between the control group and the intervention group

\begin{tabular}{|c|c|c|c|c|c|c|c|c|c|c|}
\hline & \multicolumn{2}{|c|}{ Before EVT } & \multicolumn{2}{|c|}{3 months after discharge } & \multicolumn{3}{|c|}{ Main effect } & \multicolumn{3}{|c|}{ interaction } \\
\hline & $\begin{array}{l}\text { Control } \\
\text { group } \\
(n=15)\end{array}$ & $\begin{array}{l}\text { Interven- } \\
\text { tion group } \\
(\mathrm{n}=15)\end{array}$ & $\begin{array}{l}\text { Control } \\
\text { group } \\
(n=15)\end{array}$ & $\begin{array}{l}\text { Intervention } \\
\text { group }(n=15)\end{array}$ & $\begin{array}{c}\mathrm{F} \\
\text { value }\end{array}$ & $\begin{array}{c}\mathrm{p} \\
\text { value }\end{array}$ & $\begin{array}{c}\text { Effect } \\
\text { size } \\
\left(\eta^{2}\right)\end{array}$ & $\begin{array}{c}\mathrm{F} \\
\text { value }\end{array}$ & $\begin{array}{c}\mathrm{p} \\
\text { value }\end{array}$ & $\begin{array}{c}\text { Effect } \\
\text { size } \\
\left(\eta^{2}\right)\end{array}$ \\
\hline \multicolumn{11}{|l|}{ WIQ (AVG $\pm S D)$} \\
\hline Pain & $43.3 \pm 24.9$ & $34.7 \pm 23.0$ & $61.6 \pm 22.1 *$ & $83.3 \pm 21.7 * * \dagger$ & 28.5 & $<0.01$ & 0.32 & 5.86 & 0.01 & 0.07 \\
\hline distance & $33.1 \pm 30.1$ & $36.5 \pm 31.9$ & $69.7 \pm 26.8$ & $79.8 \pm 22.9$ & 26.3 & $<0.01$ & 0.32 & 0.18 & 0.66 & 0.01 \\
\hline speed & $35.4 \pm 15.2$ & $32.5 \pm 14.0$ & $55.2 \pm 20.5^{*}$ & $70.4 \pm 16.8 * * \dagger$ & 39.0 & $<0.01$ & 0.40 & 3.80 & 0.04 & 0.04 \\
\hline climbing & $40.2 \pm 25.4$ & $37.8 \pm 33.6$ & $54.4 \pm 22.4$ & $68.3 \pm 27.9$ & 8.84 & 0.01 & 0.14 & 1.17 & 0.28 & 0.02 \\
\hline total & $152.2 \pm 72.7$ & $141.5 \pm 92.8$ & $241.1 \pm 79.2$ & $301.9 \pm 70.7$ & 33.6 & $<0.01$ & 0.37 & 2.76 & 0.10 & 0.03 \\
\hline SEPA $(A V G \pm S D)$ & $46.3 \pm 21.8$ & $42.6 \pm 27.6$ & $45.4 \pm 14.4$ & $53.8 \pm 25.4 * *$ & 7.76 & 0.01 & 0.12 & 4.99 & 0.03 & 0.08 \\
\hline Vascu QOL $(\mathrm{AVG} \pm \mathrm{SD})$ & $103.0 \pm 39.5$ & $108.3 \pm 41.6$ & $133.4 \pm 28.2$ & $152.5 \pm 14.1$ & 21.0 & $<0.01$ & 0.27 & 0.68 & 0.41 & 0.01 \\
\hline
\end{tabular}

WIQ; walking impairment questionnaire, SEPA; self-efficacy for physical activity, Vascu QOL; vascular quality of life questionnaire, EVT; endovascular treatment

Wilcoxon rank-sum test: * vs. Before EVT control group $\mathrm{p}<0.05$, ** vs. Before EVT intervention group p $<0.05$, †vs. 3 months after EVT intervention group $\mathrm{p}<0.05$

successful in encouraging behavioral change in patients by setting specific steps in addition to periodically re-setting goals and providing guidance on how to achieve those goals.

Among the items that had a significant interaction with the presence or absence of the intervention, significantly higher WIQ pain and WIQ velocity scores were observed in the intervention group than in the control group 3 months after discharge. A significant increase in SEPA was also observed preoperatively and 3 months after surgery in the intervention group only. The combination of EVT and exercise therapy has been reported to improve walking distance and quality of life in both the short and long term compared to EVT and exercise therapy individually ${ }^{27)}$. Furthermore, home-based exercise therapy has also been reported to improve quality of life indicators ${ }^{28)}$. McDermott et $\mathrm{al}^{16)}$ reported a $34 \%$ increase in WIQ distance score and a $32 \%$ increase in WIQ speed score from baseline after 6 months of home exercise. The present study showed similar results. Increased SEPA and Vascu QOL have been reported in patients with PAD along with improved walking ability $^{29,30)}$. Quality of life and self-efficacy of the patients in this study may also have improved as the number of steps increased, although an increase in the number of steps is not equal to an increase in walking ability.

There are several limitations to this study. First, due to insufficient numbers of patients, it was not possible to perform a proper statistical analysis of changes in the number of steps walked. Therefore, the effect sizes were tested retrospectively, but the effect sizes for the primary outcome were moderate. Secondly, participation was also very limited because some subjects did not consent to the study and some withdrew from the study. Therefore, the majority of participants are considered to be highly compliant with the exercise. Third, several patients in the intervention group failed to achieve the initial goal of 1300 steps/day from their pre-hospitalization step count. Because these patients were included in the analysis, not all subjects in the intervention group were able to perform the home-based exercise targeted in this study. Finally, the results show only short-term effects, and the medium- to long-term effects are unknown.

\section{Conclusion}

In summary, home-based exercise therapy using physical activity indices improves number of steps and quality of life for PAD patients after EVT. Our findings suggest that providing personalized, home-based exercise therapy may help PAD patients with PAD to develop regular physical exercise habits after discharge from hospital.

Acknowledgement: The authors acknowledge the work of past and present members of our laboratory and department of rehabilitation, and Dr. Ishida. The authors would like to thank Enago (www.enago.jp) for the English language review.

Conflict of Interest: The authors declare that we have no competing interests.

\section{References}

1) Norgren L, Hiatt WR, et al.: Inter-Society Consensus for the Management of Peripheral Arterial Disease (TASC II). J Vasc Surg. 2007; 45: 5-67.

2) Fowkes FG, Rudan D, et al:: Comparison of global estimates of prevalence and risk factors for peripheral artery disease in 2000 and 2010: a systematic review and analysis. Lancet. 2013; 382 (9901): 1329-1340.

3) Otsuka S, Morisawa T, et al.: Clinical importance of change in 
physical activity after endovascular treatment combined with exercise training in patients with peripheral arterial disease. Heart Vessels. 2017; 32: 143-148.

4) McDermott MM, Liu K, et al.: Measuring physical activity in peripheral arterial disease: a comparison of two physical activity questionnaires with an accelerometer. Angiology. 2000; 51: 91100.

5) Lauret GJ, Fokkenrood HJ, et al.: Physical activity monitoring in patients with intermittent claudication. Eur J Vasc Endovasc Surg. 2014; 47: 656-663.

6) Gardner AW, Montgomery PS, et al.: Patterns of ambulatory activity in subjects with and without intermittent claudication. J Vasc Surg. 2007; 46: 1208-1214.

7) Leeper NJ, Myers J, et al.: Exercise capacity is the strongest predictor of mortality in patients with peripheral arterial disease. $\mathrm{J}$ Vasc Surg. 2013; 57: 728-733.

8) Garg PK, Liu K, et al.: Physical activity during daily life and functional decline in peripheral arterial disease. Circulation. 2009; 119: 251-260.

9) Matsuo T, Sakaguchi T, et al:: Effect of in-hospital physical activity on cardiovascular prognosis in lower extremity bypass for claudication. J Phys Ther Sci. 2015; 27: 1855-1859.

10) Abaraogu UO, Dall PM, et al.: The effect of structured patient education on physical activity in patients with peripheral arterial disease and intermittent claudication: a systematic review. Eur J Vasc Endovasc Surg. 2017; 54: 58-68.

11) Burton NW, Ademi Z, et al.: Efficacy of brief behavioral counselling by allied health professionals to promote physical activity in people with peripheral arterial disease (BIPP): study protocol for a multi-center randomized controlled trial. BMC Public Health. 2016; 16: 1148.

12) Writing Committee Members, Gerhard-Herman MD, et al. : AHA/ACC guideline on the management of patients with lower extremity peripheral artery Disease: executive summary. Vasc Med. 2016; 22: 1-43.

13) Schulte KL, Hardung D, et al.: Real-world outcomes of endovascular treatment in a non-selected population with peripheral artery disease - prospective study with 2-year follow-up. Vasa. 2019; 48: 433-441.

14) McDermott MM, Guralnik JM, et al.: Unsupervised exercise and mobility loss in peripheral artery disease: a randomized controlled trial. JAHA. 2015; 4: e001659.

15) Gardner AW, Parker DE, et al.: Efficacy of quantified homebased exercise and supervised exercise in patients with intermittent claudication: a randomized controlled trial. Circulation. 2011; 123: 491-498.

16) McDermott MM, Liu K, et al.: Home-based walking exercise intervention in peripheral artery disease: A randomized clinical trial. JAMA. 2013; 310: 57-65.
17) Eivind A and Einar Y: Reliability of Objectively Measured Sedentary Time and Physical Activity in Adults. PLOS ONE. 2015 20; 10: e0133296. doi: 10.1371/journal.pone.0133296.

18) Hiatt WR, Hirsch AT, et al.: Clinical trials for claudication. Assessment of exercis performance, functional status, and clinical end points. Vascular Clinical Trialists. Circulation. 1995; 92 : 614-621.

19) Ewart CK, Taylor CB, et al.: Effects of early postmyocardial infarction exercise testing on self-perception and subsequent physical activity. Am J Cardiol. 1983; 51: 1076-1080.

20) Morgan MB, Crayford T, et al.: Developing the Vascular Quality of Life Questionnaire: a new disease-specific quality of life measure for use in lower limb ischemia. J Vasc Surg. 2001; 33: 679-687.

21) Bhatt DL, Steg PG, et al.: International prevalence, recognition, and treatment of cardiovascular risk factors in outpatients with atherothrombosis. JAMA. 2006; 295: 180-189.

22) National Health and Nutrition Examination Survey in Japan (2013). [cited 2018 Sep. 15]; Available from: http://www.mhlw. go.jp/stf/houdou/0000067890.html.

23) Gardner AW, Parker DE, et al:: Step-monitored home exercise improves ambulation, vascular function, and inflammation in symptomatic patients with peripheral artery disease: a randomized controlled trial. JAHA. 2014; 3: e001107.

24) Chang P, Nead KT, et al.: Effect of physical activity assessment on prognostication for Peripheral artery disease and mortality. Mayo Clin Proc. 2015 Mar; 90: 339-45.

25) Gardner AW, Ritti-Dias RM, et al.: Daily ambulatory activity monitoring in patients with peripheral artery disease. Phys Ther Rev. 2010; 15: 212-223.

26) Bruno RC, Breno QF, et al.: Are the Barriers for Physical Activity Practice Equal for All Peripheral Artery Disease Patients? Arch Phys Med Rehabil. 2015; 96: 248-252.

27) Kruidenier LM, Nicolai SP, et al: : Additional Supervised Exercise Therapy After a Percutaneous Vascular Intervention for Peripheral Arterial Disease: A Randomized Clinical Trial. J Vasc Interv Radiol. 2011; 22: 961-968.

28) Gardner AW: Exercise rehabilitation for peripheral artery disease: an exercise physiology perspective with special emphasis on the emerging trend of home-based exercise. Vasa. 2015; 44: 405-417.

29) Spronk S, Bosch JL, et al.: Intermittent claudication: clinical effectiveness of endovascular revascularization versus supervised hospital-based exercise training - randomized controlled trial. Radiology. 2009; 250: 586-595.

30) Lundgren F, Dahllöf AG, et al.: Intermittent claudication-surgical reconstruction or physical training? A prospective randomized trial of treatment efficiency. Ann Surg. 1989; 209: 346-355. 